Supporting information for

\title{
Layered Structure Formed in a Block Codendrimer Caused by Intermolecular Hydrogen Bonds
}

Fei Yuan', Wei Wang, Miao Yang, Xinjun Zhang, Jinying Li, Hong Li, Binglin He, Britt Minch, Günter Lieser, Gerhard Wegner

Experimental Section. All chemicals were purchased from major chemical suppliers as purity grade and used without further purification. 2,2-bis-(hydroxymethyl)propionicacid, $\quad N, N^{\prime}$-dicyclohexylcarbodiimide (DCC), p-toluenesulfonic acid (TsOH), 4-(Dimethylamino) pyridine (DMAP) (99\%) were purchased from Acros. 3,5-dihydroxybenzyl alcohol and Dowex $\mathrm{H}^{+} 50 \mathrm{~W}-\mathrm{X} 2$ ion-exchange resin were purchased from Aldrich.

\section{Characterization Methods of Chemical Structure and Physical} Property of Codendrimer.

(i) NMR. ${ }^{1} \mathrm{H}$ NMR and ${ }^{13} \mathrm{C}$ NMR spectra were recorded in $\mathrm{CDCl}_{3}$ (D 99.0\% + TMS 0.03\%, CIL) on a Bruker AM-400 spectrometer at 400.13 $\mathrm{MHz}$ at room temperature. All $\delta$ values were given in ppm downfield from tetramethylsilane (TMS).

(ii) Matrix-assisted Laser Desorption Ionization Time-of-flight Spectroscopy. MALDI-TOF mass spectra were measured with a Bruker Reflex II TOF spectrometer using a $337 \mathrm{~nm}$ nitrogen laser with a dithranol matrix.

(iii) Differential Scanning Calorimetry. DSC was measured on a Perkin-Elmer Diamond DSC with heating and cooling rates of $10{ }^{\circ} \mathrm{C} / \mathrm{min}$ while purging with nitrogen with a flow rate of $20 \mathrm{~mL} / \mathrm{min}$.

(iv). Fourier Transform Infrared spectra. For RT transmission FTIR measurements a Nicolet 730 FTIR spectrometer was used. For the 
transmission FTIR spectra with temperature were obtained in transmission on Nicolet MAGNA-IR 850 spectrometer Series II. The samples were examined in the solid state, as $\mathrm{KBr}$ pellets.

(v). X-ray Scattering System. 2D-wide angle X-ray diffraction measurements were performed with a standing anode $2.2 \mathrm{~kW}$ with a pinhole collimation and a 2D-detector with a beam diameter of ca. $0.3 \mathrm{~mm}$. An $\mathrm{X}$-ray mirror from osmic company.

Synthesis of $\boldsymbol{g} 3$-PBE- $\boldsymbol{b}$-g3-PMPA (1). g3-PMPA-COOH (0.1724 g, 0.16 $\mathrm{mmol})$, and $0.0005 \mathrm{~g}(0.15 \mathrm{mmol})$ DPTS were mixed in $5 \mathrm{ml}$ of dry $\mathrm{CH}_{2} \mathrm{Cl}_{2}$. After the reaction flask was flushed with argon, $0.0006 \mathrm{~g}(0.225$ mmol) of DCC was added. The reaction was stirred at room temperature for $15 \mathrm{~h}$ under argon. Once the reaction was complete the DCC-urea was filtered off and washed with a small volume of $\mathrm{CH}_{2} \mathrm{Cl}_{2}$. The crude product was purified by column chromatography on silica gel, using a gradient elution system (3:2 hexane/ethyl acetate to 1:1 hexane/ethyl acetate) to give 1 as a colorless glass-like solid $(0.2901 \mathrm{mg}, 75.5 \%) .{ }^{1} \mathrm{H}$ NMR $\left(\mathrm{CDCl}_{3}\right): \delta$ 1.03 (s, $\left.12 \mathrm{H}, \mathrm{CH}_{3}\right), 1.14$ (s, $\left.6 \mathrm{H}, \mathrm{CH}_{3}\right), 1.19$ (s, 3H, $\left.\mathrm{CH}_{3}\right), 1.24(\mathrm{~s}, 12 \mathrm{H}$, $\left.\mathrm{CH}_{3}\right), 1.29\left(\mathrm{~s}, 12 \mathrm{H}, \mathrm{CH}_{3}\right), 3.49\left(\mathrm{~d}, 8 \mathrm{H}, \mathrm{CH}_{2} \mathrm{O}\right), 4.03\left(\mathrm{~d}, 8 \mathrm{H}, \mathrm{CH}_{2} \mathrm{O}\right), 4.20(\mathrm{~s}$, $\left.12 \mathrm{H}, \mathrm{CH}_{2} \mathrm{O}\right), 4.95\left(\mathrm{~s}, 12 \mathrm{H}, \mathrm{PhCH}_{2} \mathrm{O}\right), 5.00\left(\mathrm{~s}, 16 \mathrm{H}, \mathrm{PhCH}_{2} \mathrm{O}\right), 5.09(\mathrm{~s}, 2 \mathrm{H}$, $\left.\mathrm{ArCH}_{2} \mathrm{O}\right), 6.49(\mathrm{~m}, 9 \mathrm{H}, \mathrm{ArH}), 6.63(\mathrm{~d}, 12 \mathrm{H}, \mathrm{ArH}), 7.31-7.41$ (m, 40H, $\mathrm{PhH}$ ); ${ }^{13} \mathrm{C} \mathrm{NMR}\left(\mathrm{CDCl}_{3}\right): \delta 17.6,18.5,22.1,25.1,25.6,33.9,42.0,46.7,46.8$, 49.1, 64.9, 65.9, 66.0, 70.0, 70.1, 98.0, 101.6, 106.4, 106.5, 127.5, 128.0, 128.5, 136.7, 137.6, 139.0, 139.2, 160.1, 171.8, 173.4. Calcd: $\mathrm{m} / \mathrm{z}$ for $\mathrm{C}_{152} \mathrm{H}_{164} \mathrm{O}_{36}[\mathrm{M}]^{+}=2565.1$ Found: MALDI-TOF: $[\mathrm{M}+\mathrm{Ag}]^{+}=2672.8$.

Synthesis of $\boldsymbol{g} 3$-PBE- $b$-g3-PMPA $(\mathbf{O H})_{8}$ (2). Compound $1(0.2000 \mathrm{~g}$, $0.077 \mathrm{mmol}$ ) was dissolved in $2 \mathrm{ml}$ of methanol and $6 \mathrm{ml}$ of $\mathrm{CH}_{2} \mathrm{Cl}_{2}$. A 
small amount of a Dowex $\mathrm{H}^{+}$resin was added. The reaction mixture was stirred for $24 \mathrm{~h}$ at room temperature. When the reaction was complete, the reaction mixture was gravity filtered to remove the Dowex $\mathrm{H}^{+}$resin. The resin was carefully washed with $\mathrm{CH}_{2} \mathrm{Cl}_{2}$. The crude product was purified by column chromatography on silica gel, using a gradient elution system (1:1 hexane/ethyl acetate to 1:2 hexane/ethyl acetate) to give $\mathbf{2}$ as a colourless glass-like solid $(0.1687 \mathrm{mg}, 90.1 \%) .{ }^{1} \mathrm{H} \mathrm{NMR}\left(\mathrm{CDCl}_{3}\right): \delta 1.03(\mathrm{~s}, 12 \mathrm{H}$, $\left.\mathrm{CH}_{3}\right), 1.21$ (s, 6H, $\left.\mathrm{CH}_{3}\right), 1.27$ (s, $\left.3 \mathrm{H}, \mathrm{CH}_{3}\right), 3.62\left(\mathrm{~d}, 8 \mathrm{H}, \mathrm{CH}_{2} \mathrm{O}\right), 3.71(\mathrm{~d}, 8$ $\left.\mathrm{H}, \mathrm{CH}_{2} \mathrm{O}\right), 4.27\left(\mathrm{~m}, 12 \mathrm{H}, \mathrm{CH}_{2} \mathrm{O}\right), 4.96\left(\mathrm{~s}, 12 \mathrm{H}, \mathrm{PhCH}_{2} \mathrm{O}\right), 5.03(\mathrm{~s}, 16 \mathrm{H}$, $\left.\mathrm{PhCH}_{2} \mathrm{O}\right), 5.10\left(\mathrm{~s}, 2 \mathrm{H}, \mathrm{ArCH}_{2} \mathrm{O}\right), 6.55(\mathrm{~m}, 9 \mathrm{H}, \mathrm{ArH}), 6.67(\mathrm{~d}, 12 \mathrm{H}, J=$ $3 \mathrm{~Hz}, \mathrm{ArH}), 7.32-7.43(\mathrm{~m}, 40 \mathrm{H}, \mathrm{PhH}) ;{ }^{13} \mathrm{C} \mathrm{NMR}\left(\mathrm{CDCl}_{3}\right): \delta 17.3,18.0,46.9$, 50.0, 65.1, 66.3, 67.3, 67.7, 67.8, 70.4, 102.0, 102.4, 106.8, 107.8, 127.7, 128.1, 128.7, 137.0, 137.7, 139.3, 139.5, 160.4, 172.2, 172.6, 175.2. Calcd: $m / z$ for $\mathrm{C}_{140} \mathrm{H}_{148} \mathrm{O}_{36}[\mathrm{M}]^{+}=2405.0$ Found: MALDI-TOF: [M+Ag] ${ }^{+}=2513.8$.

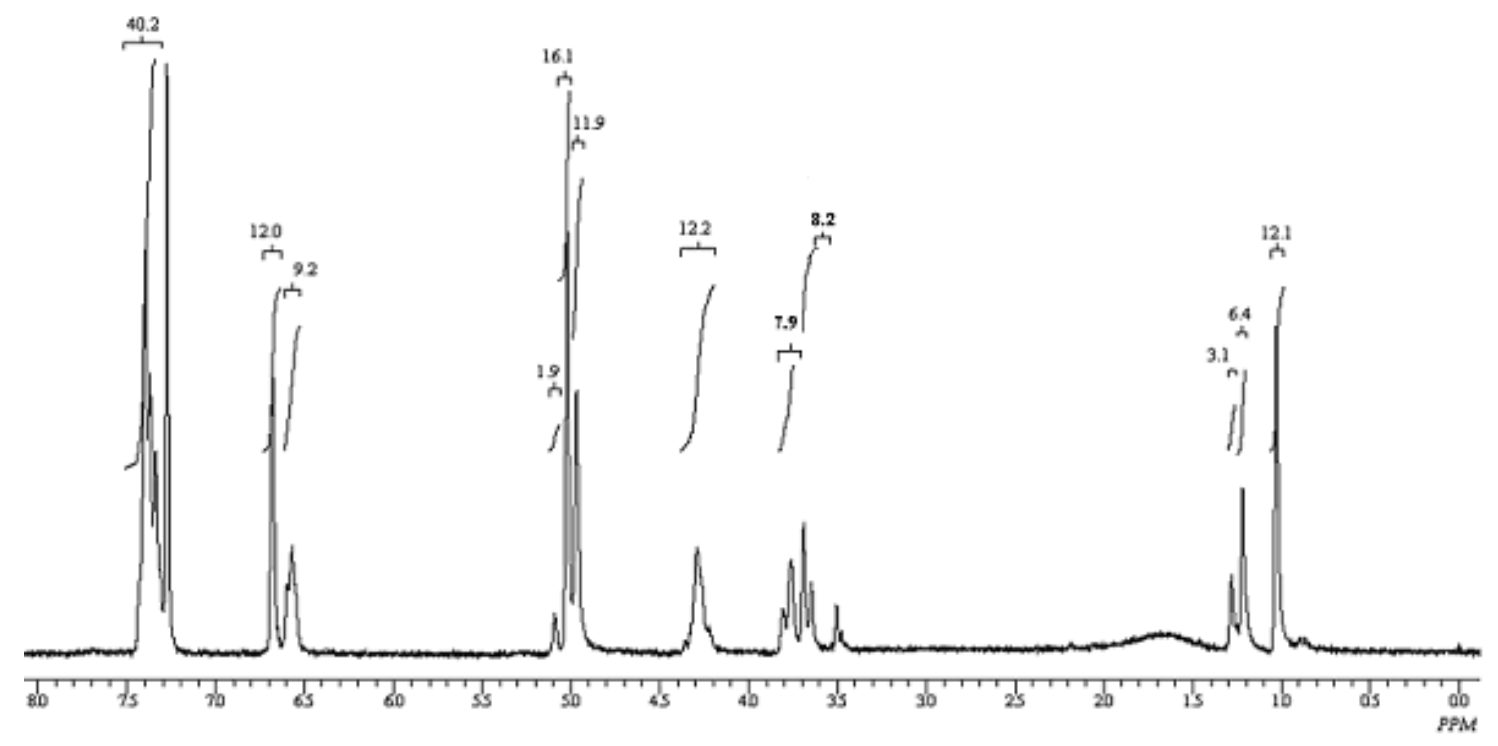

Figure S1. ${ }^{1} \mathrm{H}$ NMR spectra of 2. 


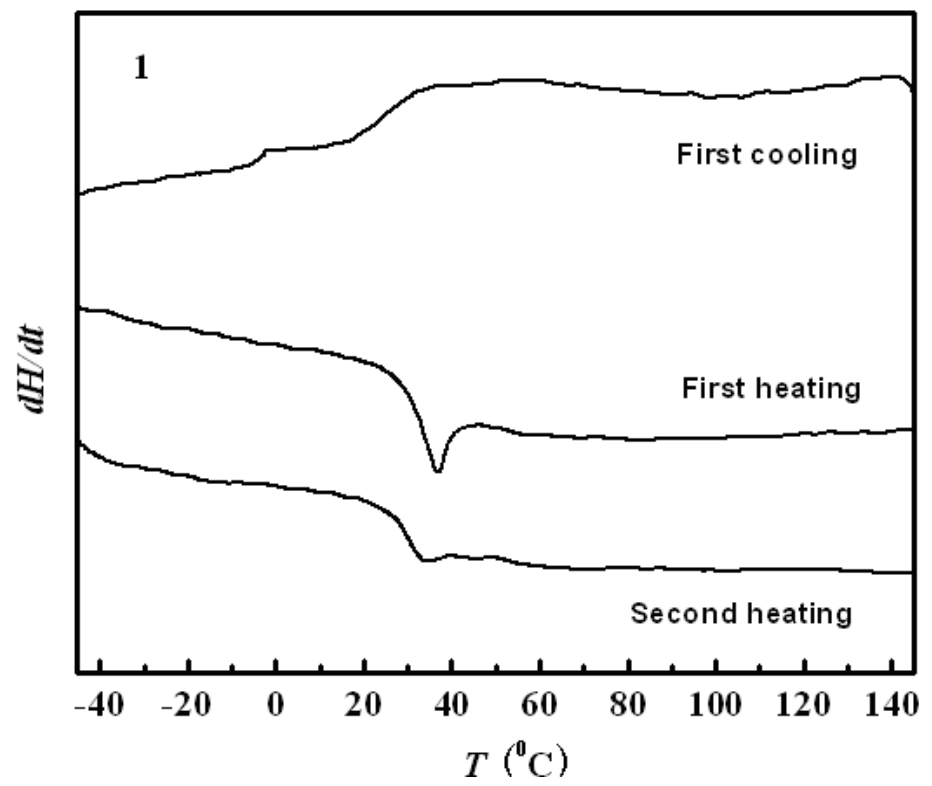

Figure S2. DSC of $\mathbf{1}$. The measurements were conducted at a rate of $10 \mathrm{~K} /$ min under a nitrogen atmosphere.

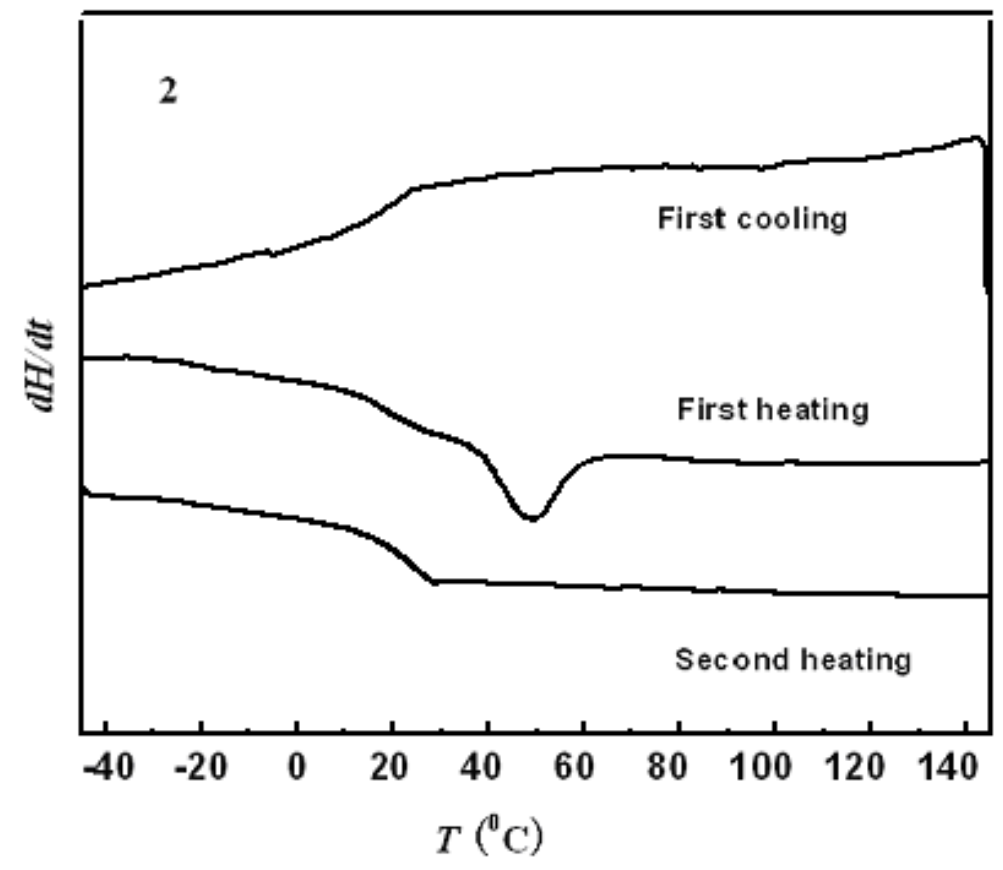

Figure S3. DSC of 2. The measurements were conducted at a rate of $10 \mathrm{~K} /$ min under a nitrogen atmosphere. 


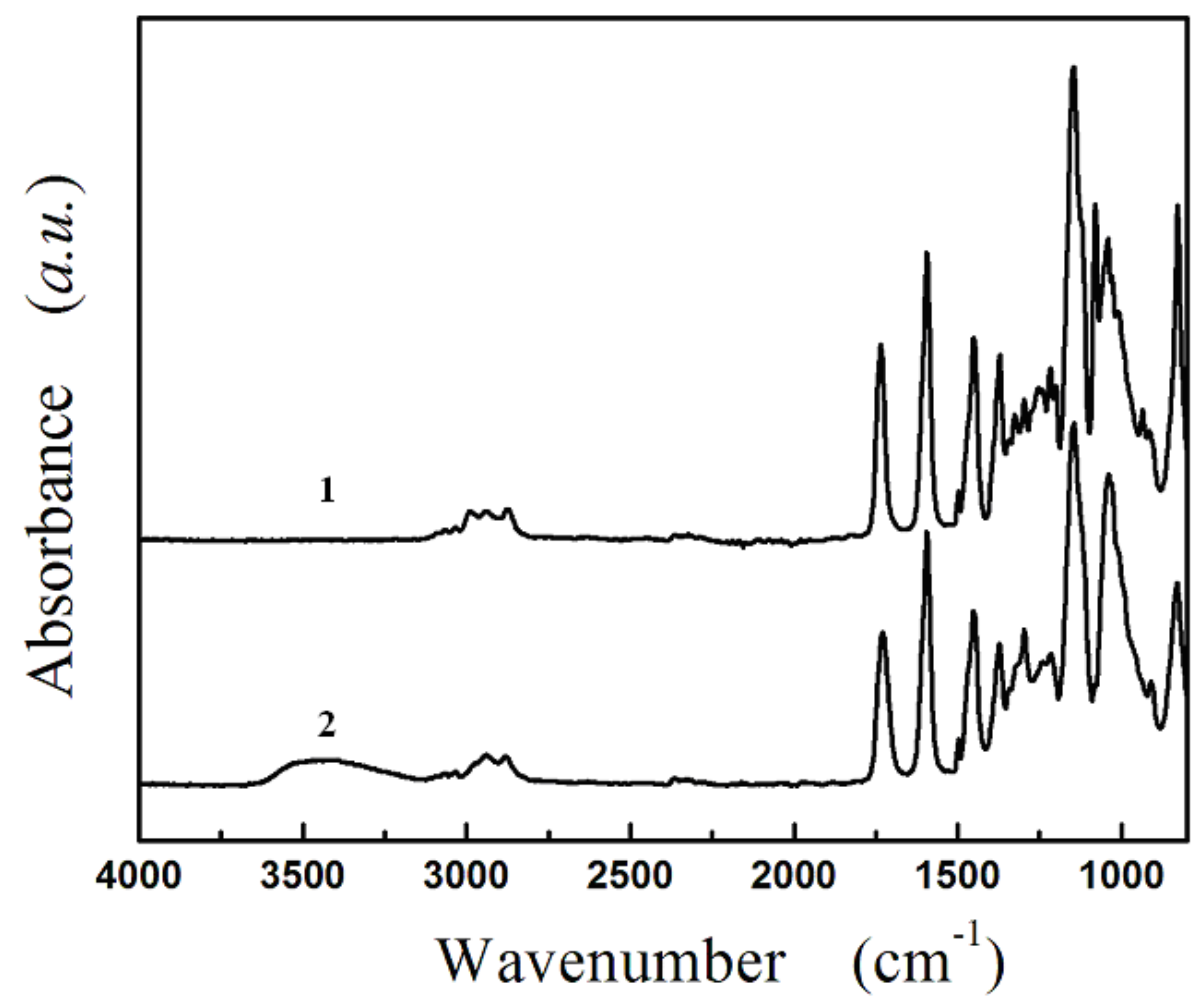

Figure S4. FT-IR spectra of $\mathbf{1}$ and $\mathbf{2}$.

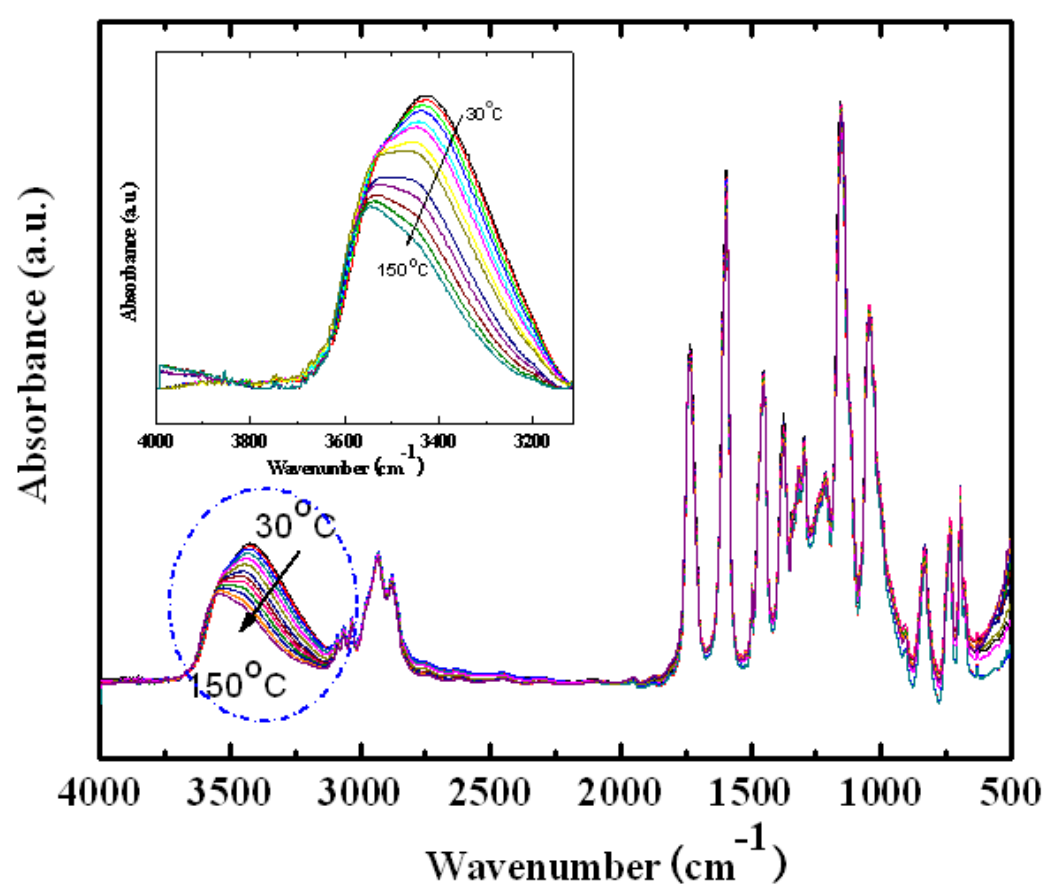

Figure S5. Temperature dependent FT-IR spectra of 2 from $30^{\circ} \mathrm{C} \sim 150^{\circ} \mathrm{C}$, $10^{\circ} \mathrm{C}$ as a step. 


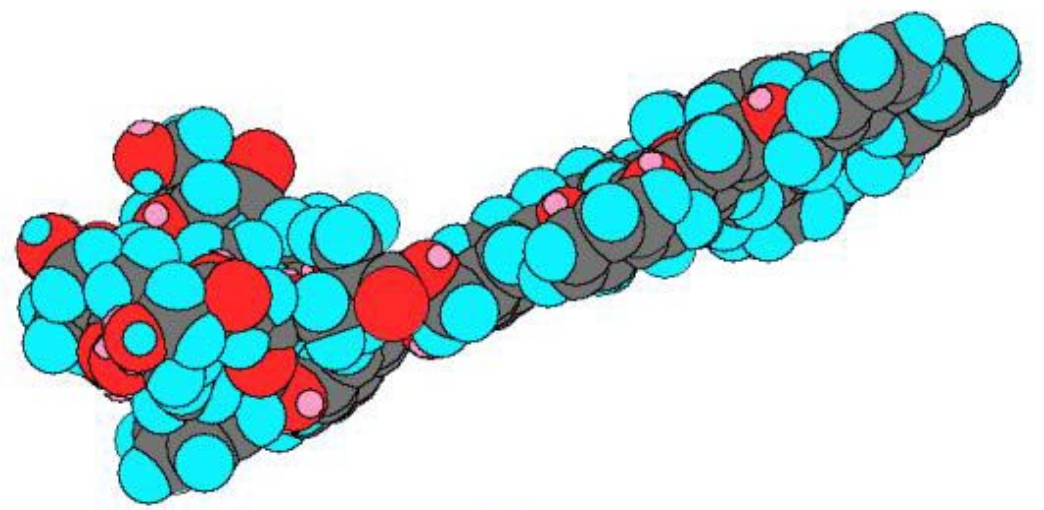

(A)

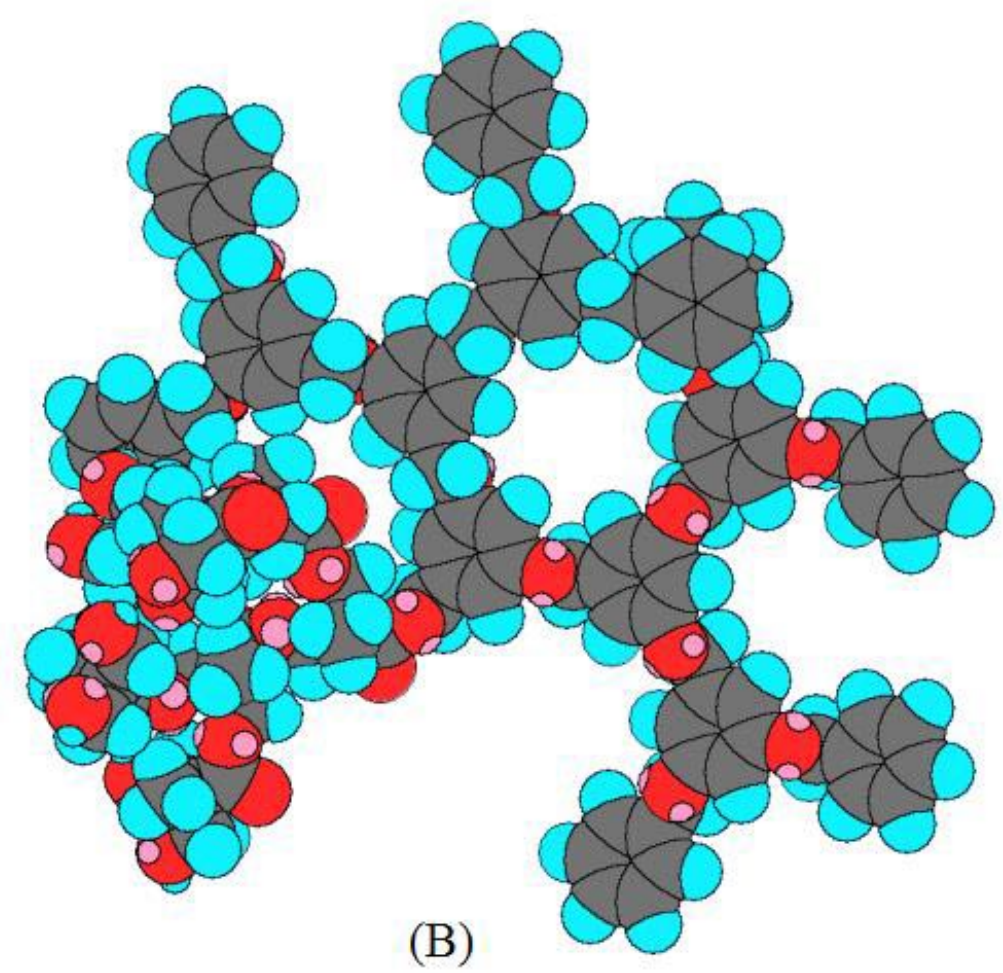

Figure S6. A simple computer simulation of the single block codendrimer 2 using Chemdraw software. (A) Side view and (b) Top view. The left part is the $g 3-\mathrm{PMPA}(\mathrm{OH})_{8}$ block and the right part is the $g 3$-PBE block. 